\title{
Téoros
}

Revue de recherche en tourisme

\section{Femmes et produits touristiques spécifiques}

\section{Peu de sollicitation directe}

\section{Marie-Anne Poussart}

Volume 16, numéro 3, automne 1997

Femmes et tourisme

URI : https://id.erudit.org/iderudit/1073315ar

DOI : https://doi.org/10.7202/1073315ar

Aller au sommaire du numéro

Éditeur(s)

Université du Québec à Montréal

ISSN

0712-8657 (imprimé)

1923-2705 (numérique)

Découvrir la revue

Citer cet article

Poussart, M.-A. (1997). Femmes et produits touristiques spécifiques : peu de sollicitation directe. Téoros, 16(3), 23-24. https://doi.org/10.7202/1073315ar d'utilisation que vous pouvez consulter en ligne.

https://apropos.erudit.org/fr/usagers/politique-dutilisation/ 


\section{FEMMES ET PRODUITS TOURISTIQUES SPÉCIFIQUES}

\section{PEU DE SOLLICITATION DIRECTE}

Marie-Anne Poussart, journaliste et rédactrice

Les vacances-santé sont sans doute le produit touristique que l'on associe le plus directement aux femmes. Pourtant, s'il est encore vrai qu'au Québec la clientèle de ces centres où l'on offre soins pour le corps et activités de détente est majoritairement féminine, les relais santé québécois tentent actuellement une percée du côté des hommes. * A la fin des années 80 et au début des années 90 , il nous a surtout fallu travailler à faire connaitre le concept des vacances-sante. Tout naturellement, ce sont d'abord les fennes qui s'y sont intéressées. Mais maintenant que le public québécois connaît mieux ce que nous offrons, nous tentons d'attirer les hommes vers nos centres", explique Lucie Octeau, directrice de l'Association des relais-santé du Québec.

Exception faite des centres de santé, il est plutôt étonnant de constater à quel point les Québécoises sont peu sollicitées par des produits touristiques spécifiques. En effet, contrairement aux États-Unis où les voyagistes et établissements hôteliers s"arrachent cette clientèle, le Québec offre aux femmes relativement peu d'activités et services spécifiques.

Parmi les entreprises ayant pris l'initiative de répondre de façon particulière aux besoins ou attentes des femmes, on compte plusieurs centres de ski. Plus d'une vingtaine d'entre eux, répartis à travers la province, offrent entre autres un service de halte-garderie qui permet aux mères de jeunes enfants de pratiquer leur sport préfété en toute quiétude. Quelques stations ont même mis sur pied des programmes s'adressant uniquement aux femmes. C'est notamment le cas de Tremblant, qui, depuis trois ans, propose des leçons d'initiation à des groupes constitués uniquement de femmes. Offertes une fois par semaine, ces leçons, données par des monitrices plutôt que des moniteurs, visent a faciliter l'apprentissage des femmes. "L'esprit de compétition est beaucoup plus grand chez les hommes que chez les femmes\%, explique Isabelle Blanchet, du service des communications de la station de ski. "Nous voulions permettre aux non-initiées d'apprendre sans être intimidées. L'intérêt qu'elles ont manifesté jusqu'ici pour ces leçons nous ont convaincu de leur pertinence. $x$.

L'hôtel Le Chantecler de Sainte-Adèle a lui aussi récemment développé un produit particulier pour les femmes. Depuis 1'été 1997, il offre aux parents uniques - qui sont majoritairement des femmes - l'occasion de passer une fin de semaine de plein air abordable avec leur jeune. L'été, le forfait donne accès à la plage et aux courts de tennis, en plus de permettre l'utilisation des embarcations nautiques. Encore une fois, le produit a été développé pour répondre à un besoin qui se faisait sentir. «De plus en plus de femmes êlèvent seules leur enfant. Il nous apparaissait nécessaire de leur offrir un produit qui corresponde spécifiquement à leurs besoinsw, résume-t-on, au Chantecler.

À l'hôtel Loews Le Concorde, de Québec, tout un étage est par ailleurs réservé aux femmes, et ce depuis 10 ans. Il s'agit du seul hôtel québécois à offrir ce service.
L'hôtel Reine Elizabeth, de Montréal, a déjà eu des chambres réservées aux voyageuses, mais une série de plaintes ont poussé la direction de l'établissement à revoir sa politique. En effet, certaines femmes étaient contre leur ghettoïsation et d'autres craignaient pour leur sécurité.

\section{LES FEMMES D'AFFAIRES}

Et qu'en est-il des femmes d'affaires? Au Canada, cette clientèle demeure, elle aussi, encore peu sollicitée directement. En effet, si les produits et services offerts à la clientèle d'affaires sont courants et de plus en plus développés (taux corporatifs dans les grands hôtels, chambres équipées de prises téléphoniques multiples pour permettre l'utilisation de modems, etc.), ceux visant directement les professionnelles sont beaucoup plus rares.

Par contre, certains hôtels, comme l'établissement Minto Place Suite Hôtel d'Ottawa, offrent une série de services visant à répondre aux besoins particuliers des femmes d'affaires. Souvent appelées à voyager seules, ces dernières apprécient de façon particulière les endroits où elles se sentent en sécurité. D'après Miranda Davies et Natania Jansz, auteures du guide Women Travel (1990), la peur d'être attaquée ou agressée constitue la plus grande crainte des femmes qui voyagent seules ou entre amies. Selon les auteures, cette peur limite non seulement les femmes dans leurs activités mais affecte également plusieurs aspects de leurs séjours à l'étranger. C'est une des raisons qui a poussé Place Minto Suite Hôtel à élaborer un système visant â assurer une sécurité hors pair à ses clientes. Ainsi, les clefs des suites de l'établissement ne portent aucun 
numéro et sont magnétisées plutôt que taillées de façon traditonnelle dans du métal. Grâce au système de verrouillage Marlock, les clientes de l'hôtel sont par ailleurs assurées d'être les seules à pouvoir pénétrer dans leur suite. De plus, des gardes de sécurité arpentent les différents étages de l'établissement 24 heures par jour. Enfin, dans le stationnement de l'hôtel, on a également prévu des postes d'assistance en cas d'urgence, à partir desquels il est possible de parler à un agent de sécurité en tout temps.

Quant à l'aménagement des suites, il a lui aussi été pensé pour rendre le séjour des femmes d'affaires plus agréable. Dans plusieurs suites, on retrouve entre autres une cuisine entièrement équipée (avec poèle, réfrigérateur, micro-ondes, lavevaisselle, batterie de cuisine, ustensiles, etc.). "Les femmes d'affaires sont nombreuses à les trouver pratiques. Plusieurs d'entre elles préferent se cuisiner ellesmêmes leurs repas plutôt que de sortir et manger seules au restaurant», souligne Charles Nadeau, directeur du marketing et des ventes de l'hôtel.

Dans son mémoire intitule La femme d'affaires: un créneau pour l'hotellerie, Caroline Lipovsky (1986a) explique que les besoins des femmes et des hommes d'affaires sont largement similaires, mais qu'un établissement hôtelier gagne tout de même à répondre aux besoins plus spécifiques des femmes. «Parmi les besoins tangibles (des femmes), on peut noter un sèche-cheveux, un fer à repasser, une penderie suffisamment haute, des cintres en grande quantité - y compris des cintres à jupes -, un bon éclairage dans la salle de bain et enfin un room-services, ecrit-elle (1986a: 181). En ce qui a trait aux besoins intangibles, elle souligne l'importance des points suivants: aune sécurité garantie, un bar plaisant où l'on puisse se détendre en toute tranquillité et éventuellement un salon de coiffure ou de beauté.m Lipovsky fait par ailleurs remarquer que les femmes d'affaires wne veulent pas de traitement particuliero, mais «qu’à défaut d'exiger certains services qui lui facilitent la vie à l'hồtel, la femme d'affaires réclame avant tout un service sans reproche qui lui permette d'accomplir efficacement sa mission professionnelle.s (1986b;6)

\section{DU CÔTÉ AMÉRICAIN}

Alors que les entreprises qui oeuvrent dans le milieu touristique québécois hésitent à définir leur produit comme étant spécifiquement destiné aux femmes, préférant cibler une clientèle plus large, plusieurs voyagistes américains ont fait de la clientèlé féminine leur préoccupation première. D'ailleurs, le phénomène des entreprises se specialisant en voyages pour femmes seulement est tel qu'Arthur Frommer y consacre toute une section dans la plus récente édition de son livre New World of Travel (1996). On y apprend entre autres que Woodswomen, qui a son siège social à Minneapolis, est le plus important et le plus ancien des grossistes américains oeuvrant dans le domaine du tourisme au féminin.

En lisant la documentation du voyagiste, on se rend compte de la diversité des produits offerts: voyages d'equitation dans le Wisconsin, de cyclotourisme en Nouvelle-Zélande, d'escalade au Népal, de canot-camping au Minnesota, de ski au Colorado, etc. L'organisme, qui est sans but lucratif et existe depuis plus de 20 ans, vise avant tout à offrir aux femmes une expérience unique de voyage. On souligne aux intéressées qu'elles prendront part à des activitếs organisées en fonction de leurs besoins spécifiques, qu'elles apprendront puisque chaque excursion/expédition se veut en partie éducative et qu'elles se reposeront puisqu'elles n'auront à se préoccuper d'aucun des détails techniques liés à l'organisation de tels voyages (réservations, planification des repas, sécurité, ete.). En 1995 , plus de 800 femmes, âgées de 18 à 70 ans, ont participé aux quelques 70 voyages de courte et de longue durée qui étaient offerts.

Parmi les autres grossistes américains en voyages pour femmes, on retrouve Roots and Wings Excursions, situé en Virginie, qui se spécialise dans l'organisation de voyages pour mères et filles, un créneau qui reste très peu exploité. Au moins deux autres, Artemis Sailing Charters, situé en Idaho, et Womanship, situé au Maryland, organisent quant à eux des vacances en voilier pour femmes seulement. Celles qui optent pour les produits de ces entreprises apprennent ainsi, tout en voyageant, les rudiments d'un sport encore pratiqué majoritairement par des hommes. C'est d'ailleurs pour permettre aux femmes de réellement diriger l'embarcation et de superviser l'ensemble des opérations que les organisatrices n'acceptent aucun homme à bord lorsque des groupes partent en croisière. Offrir un produit qui s'adresserait avant tout à une clientèle homosexuelle n'est done pas le but de ces entreprises.
Au Canada, les voyagistes tournés vers une clientèle féminine sont peu nombreux. On retrouve en Colombie-Britannique au moins deux groupes, dont Ocean West Expeditions Ltd., qui organise à l'occasion des séjours de tourisme d'aventure pour des groupes de femmes sur la Cote Ouest. Parhways Canada Tours organise quant à lui des expéditions en canot pour femmes seulement dans la région des Lacs Bowron. Canada's Canoe Adventures, basé à Merrickville, en Ontario, propose aussi une gamme d'expéditions en canot pour femmes seulement. Quelques voyagistes américains, tel Woodswomen, organisent aussi des voyages au Canada, sans toutefois y avoir de bureaux. L'internet s'avère alors un excellent outil pour en connaître davantage sur leurs produits.

La propriétaire d'une petite auberge en Colombie-Britannique, le Avonlea West Island Retreat for Women, a par ailleurs choisi de faire des femmes ses uniques clientes. *En tant que femmes, nous sommes très souvent appelées à remplir le rổle d'hotesse, mais il est rare qu'on nous offre l'occasion de nous reposer réellement. Lorsque j'ai ouvert mon établissement, je me suis dit que je nous le réservais! explique la propriétaire, Shelby Toderel, dans le site internet de l"auberge.

Il existe enfin un magazine californien s'adressant spécifiquement aux voyageuses et passionnées de voyages. Maiden Voyages Magazine, qui est publié quatre fois par année, traite au sein de ses pages de tous les aspects du tourisme au feminin. De plus, le site internet de la publication (http $/ /$ maiden-voyages.com/) comprend un répertoire détaillé des nombreux voyagistes américains offrant des produits spécifiques aux femmes.

\section{BIBLIOGRAHIE}

Davies, Miranda et Natania Jansz (1990), Women Travel - A Real Guide Special, New York, Prentice Hall.

Frommer, Arthur (1996), New World of Travel (5th Edition), New York, MacMillan Travel

Lipovsky, Caroline (1986a), La fenme d'affai. res: un créneau pour l holellerie:, mémoire de maitrise, Centre d'études supérieures du tourisme, Université de Paris I, Panthéon Sorbonne.

Lipovsky, Caroline (1986b), «La femme d'affaires: un créneau pour tholelleries, rapport de synthèse, Centre d'études supérieures du tourisme, Université de Paris I, Panthên Sorbonne. 